\title{
DESORDENS ALIMENTARES NO DESPORTO: SITUAÇÃO ATUAL E PERSPECTIVAS FUTURAS NO ESTUDO DOS FATORES PSICOLÓGICOS ${ }^{\mathrm{I}}$
}

Luiz Silva*

Rui Gomes**

\section{Resumo}

Este trabalho procura atingir dois objetivos. Em primeiro lugar, apresenta-se a situaçâo atual da investigaçáo sobre desordens alimentares no desporto. Neste caso, salientam-se as linhas de investigação dedicadas ao estudo da prevalência destes problemas no desporto e analisam-se as diferenças entre atletas e modalidades desportivas. Dadas as dificuldades destas linhas de investigação na compreensão dos comportamentos alimentares de risco nos atletas, são avançadas outras possibilidades de desenvolvimento da investigaçáo. Assim, e enquanto segundo objetivo deste artigo, salientamos a necessidade de os estudos se dirigirem para a compreensão dos fatores psicológicos associados aos comportamentos alimentares de risco e implicados no desenvolvimento das desordens alimentares. Esta abordagem tem como vantagem adicional ajudar a prevenir estes problemas através da promoçáo das competências mentais dos atletas no sentido de resistirem melhor aos possíveis efeitos nocivos da prática desportiva, onde se inserem os problemas com a alimentação.

Palavras-chave: alimentação; desordens alimentares; psicologia do desporto.

\begin{abstract}
EATING DISORDERS IN SPORT: CURRENT STATUS AND FUTURE DIRECTIONS IN THE STUDY OF THE PSYCHOLOGICAL FACTORS
\end{abstract}

This paper focuses on two main goals. In first place, we present the current status on the research about eating disorders in sport contexts. In this case, we point out studies dedicated to the analysis of the incidence of eating disorders in sport and studies that observe the differences between

* Escola de Psicologia, Universidade do Minho, Braga, Portugal. E-mail: rgomes@psi.uminho.pt.

** Escola de Psicologia, Universidade do Minho, Braga, Portugal. E-mail:rgomes@psi.uminho.pt. 
athletes and different sports in the tendency for these problems. Second, we proposed new research directions on this subject, namely the need of analysing the psychological factors that are related with the development of eating disorders on athletes. This research approach has the advantage of helping the prevention of eating disorders on athletes through the promotion of psychological skills that protect athletes from the negative effects of sport practicing, where are included maladaptive eating behaviors. Keywords: eating disorders; eating behaviors; sport psychology.

Nos últimos 20 anos tem-se assistido a um interesse crescente pelo estudo dos comportamentos e desordens alimentares dos atletas (Byrne \& McLean, 2001). No entanto, a variedade de metodologias utilizadas e de populações desportivas analisadas não tornam ainda muito claro o verdadeiro impacto destes problemas nos desportistas.

Neste sentido, é possível constatarmos estudos mais direcionados para a análise das diferenças entre a população desportiva e não desportiva na ocorrência de desordens alimentares, a que se seguiram estudos mais direcionados para a compreensão deste tema entre diferentes tipos de desportos e atletas. Mais recentemente, temos assistido a uma maior preocupaçáo em compreender a relaçáo entre as desordens alimentares e os fatores psicológicos, sociais e ambientais onde se inserem os atletas, existindo mesmo propostas de modelos explicativos sobre as desordens alimentares em contextos desportivos.

É neste sentido que este trabalho pode ser inserido, procurando analisar a evolução da investigaçáo acerca das desordens alimentares em contextos desportivos, seguindo o percurso histórico dos trabalhos realizados e das questóes levantadas pelos autores que se dedicaram a este tema.

Mais especificamente, começamos por analisar neste trabalho a questáo de saber o que são as desordens alimentares para depois nos centrarmos apenas na análise deste tema no âmbito desportivo. $\mathrm{Na}$ aplicaçáo ao desporto, começamos por responder à questáo de saber se os atletas e o contexto desportivo representam elementos de risco para as desordens alimentares para depois questionarmos quais as características do funcionamento dos atletas que os poderão colocar em maior risco para os problemas de comportamento alimentar. Na última parte deste trabalho, procuramos questionar perspetivas futuras de estudo e alertar para as indicaçóes teóricas mais recentes sobre o estudo desta temática no desporto que têm vindo salientar a importância da compreensão dos fatores psicológicos associados às desordens alimentares nos atletas.

Com esta organização do trabalho, tentamos apoiar os investigadores interessados neste tema sobre qual a evoluçáo da investigação realizada até atualmente e em que áreas poderá ser interessante desenvolver esforços de investigação no futuro. 


\section{O que são as desordens alimentares?}

As desordens alimentares, de que são exemplo a anorexia nervosa, a bulimia nervosa e as desordens alimentares SOE (“Sem Outra Especificação”) representam condiçóes psiquiátricas com sintomas no nível comportamental (por exemplo, dieta, ingestão compulsiva), físico (por exemplo, perda de peso) e psicológico (por exemplo, preocupação com a imagem corporal) (American Psychiatric Association, APA, 2000). Estes problemas são caracterizados por distorçôes no nível percetivo e cognitivo, necessitando de tratamento clínico (American Psychiatric Association, APA, 2002).

Neste sentido, as pessoas que apresentam desordens alimentares tendem a assumir padróes alimentares patogênicos e comportamentos de controle alimentar que resultam de cogniçóes disfuncionais acerca do seu corpo bem como pensamentos que interferem no seu funcionamento diário. Esta vertente psicológica das desordens alimentares tem sido reforçada pelos autores, sugerindo-se que os problemas de comportamento alimentar resultam de atitudes, comportamentos e estratégias disfuncionais associadas a uma preocupação permanente em relação ao peso e à estética corporal (Fairburn, 1997). Deste modo, e segundo Raich (2001), as desordens alimentares referem-se, regra geral, a problemas psicológicos que se caracterizam por disfunçóes graves no comportamento de ingestão de que são exemplo a anorexia e a bulimia nervosa.

No que se refere à anorexia nervosa, esta é entendida como uma desordem alimentar que afeta principalmente jovens adolescentes do sexo feminino, que voluntariamente reduzem a ingestáo alimentar (APA, 2000). Esta tendência tem por base um medo mórbido de engordar que leva a uma perda gradual e desejada de peso, podendo chegar em casos extremos à inanição e até a morte. Do ponto de vista somático, a anorexia caracteriza-se pela desnutrição proteico-calórica e pela amenorreia (Raich, 2001).

Quanto à bulimia nervosa, esta apresenta como principal característica episódios de ingestáo alimentar incontroláveis seguidos por algum tipo de comportamento compensatório (APA, 2002). Neste caso, verifica-se que a pessoa faz uma ingestão alimentar muito superior à que seria efetuada pela maioria dos indivíduos num mesmo período de tempo. Nestas ocasióes, a pessoa tende a perceber uma sensação de falta de controle, não conseguindo evitar este comportamento disfuncional. O medo de engordar, os sentimentos de mal-estar e a sensação de perda de controle tendem a estar na origem dos comportamentos compensatórios assumidos pelo indivíduo após os períodos de ingestão compulsiva. Como exemplo destes comportamentos podem ser referidos a indução ao vômito, o uso 
abusivo de laxantes e/ou diuréticos, a prática excessiva de exercício físico, além das dietas extremas ou jejuns para evitar as consequências do comportamento alimentar excessivo (APA, 2002). Ao contrário da anorexia, a pessoa com bulimia não evidencia magreza extrema, apresentando um peso normal ou ligeiramente excessivo, o que torna a patologia mais difícil de ser diagnosticada.

Em termos da população geral, a prevalência de desordens alimentares não é muito elevada, embora se tenha de considerar os potenciais efeitos negativos sobre o funcionamento do indivíduo. Por exemplo, estima-se no DSM-IV-TR (American Psychiatric Association, 2002) uma prevalência da anorexia nervosa entre 0.5 e $1 \%$ para mulheres no final da adolescência e em jovens adultas. Já na bulimia nervosa, indicam-se valores entre $1 \%$ a $3 \%$ nas mulheres e $0.1 \%$ e 0.3\% nos homens (American Psychiatric Association, 1994). Estes problemas podem surgir em qualquer idade, embora seja difícil ocorrerem em crianças e adultos (Thompson \& Sherman, 1993). No entanto, alguns dados fornecidos pela literatura salientam a existência significativa de comportamentos alimentares desajustados em crianças e jovens como, por exemplo, a restrição alimentar, o receio de engordar, as distorções da imagem corporal, entre outros (Ricciardelli \& McCabe, 2001). Por exemplo, Schur, Sanders e Steiner (2000) verificaram, num estudo com crianças, que 50\% desejavam pesar menos e 16\% tinham assumido comportamentos destinados a baixar o peso.

\section{Estarão os atletas em risco?}

Neste nível pode-se colocar as questóes de saber se existe a possibilidade de os atletas estarem particularmente sujeitos às desordens alimentares e de se representam um grupo de risco. Segundo Cogan (2005), existem alguns fatores desportivos que, apesar de não representarem critérios de diagnóstico clínico, estão relacionados com as perturbaçóes do comportamento alimentar no desporto. São eles a ingestão compulsiva, a tríade da mulher atleta, a dependência do exercício e a anorexia atlética.

No primeiro caso, verifica-se uma ingestáo elevada de alimentos sem que seja induzido o vômito. Estes atletas têm tendência a ter excesso de peso, dado o fato de comerem mesmo não tendo fome, pois a ingestão de alimentos ajuda-os a lidar com determinadas emoçôes negativas, como a raiva, a solidão, a depressão e a tristeza. Esta problemática é de difícil detecção, uma vez que os atletas tendem a possuir necessidades calóricas diárias significativas, devido a terem um metabolismo basal elevado e um ciclo de treinos exigente. 
Em segundo lugar, a tríade da mulher-atleta refere-se a atletas que apresentam o risco de desenvolverem desordens alimentares, disfunçóes menstruais e osteoporose (Beals \& Hill, 2006). Em 1997, o Colégio Americano de Medicina Desportiva publicou uma posição sobre a tríade da mulher-atleta, definindo-a como uma síndrome preocupante que as atletas podem desenvolver no decorrer das suas carreiras (Otis, Drinkwater, Johnson, Loucks \& Wilmore, 1997). Assim, numa situação de desordem alimentar, assiste-se a um balanço energético negativo para a atleta, pois vai gastar mais energia do que a que consome. O corpo pode interpretar este balanço negativo como um processo de fome e retirar do organismo a capacidade reprodutora, resultando na cessaçáo do ciclo menstrual. Daqui resulta um decréscimo dos níveis de estrogênio e, por consequência, ocorre a amenorreia. $\mathrm{O}$ decréscimo de estrogênio no organismo, associado a uma dieta pobre em cálcio, faz com que os ossos forneçam o cálcio necessário para manter os níveis aceitáveis no organismo. Como resultado, a atleta vai perder densidade óssea, desenvolvendo osteoporose (Drinkwater, Loucks, Sherman, Sundgot-Borgen \& Thompson, 2005).

$\mathrm{O}$ terceiro fator refere-se à problemática da dependência do exercício, manifestando-se pelo nível elevado de intensidade assumido pelos atletas nos treinos bem como pelo número excessivo de horas de prática desportiva. Este padrão exagerado e compulsivo de prática desportiva pode ocorrer mesmo quando os atletas se encontram em situação de fadiga ou quando estão lesionados. De algum modo, os atletas recusam-se a aceitar estes problemas devido à possibilidade de perda de controle sobre as suas vidas, reagindo negativamente às alteraçóes das rotinas diárias. Por outro lado, e numa vertente da prática desportiva de saúde e manutenção física, verifica-se que alguns praticantes preferem realizar exercícios sozinhos, assumindo dietas rígidas e grande preocupação com o corpo. Estas características acabam por ser semelhantes às apresentadas por desportistas que sofrem de distúrbios alimentares (Morgan, 1979).

Por último, a anorexia atlética representa um estado de redução do consumo de calorias e diminuiçáo da massa corporal, tendo por base questôes relacionadas com o rendimento desportivo (Sudi et al., 2004). De acordo com Sundgot-Borgen (1993), esta condição deve ser diagnosticada nas seguintes situaçóes: (a) existência de uma perda superior a 5\% de peso atual relativamente ao esperado; (b) existência de queixas gastrointestinais; (c) falta de evidência para outras doenças ou desordens explicadoras do problema de perda de peso; (d) observaçáo de uma restrição calórica, e (e) medo extremo de ficar gordo(a). Alguns estudos têm apontado valores deste problema entre $1 \%$ para os homens e $4 \%$ para as mulheres (Sundgot-Borgen \& Torstveit, 2004). Convém realçar que esta condição em contextos desportivos é motivada por um desejo dos atletas de aumentarem os 
seus níveis de rendimento desportivo e não tanto por questôes relacionadas com a aparência física.

Tendo por base estas indicações, a investigação centrou os seus esforços no sentido de comprovar se estes quadros clínicos eram ou não significativos no âmbito desportivo.

\section{Será o desporto um contexto de risco?}

Uma das primeiras linhas de investigação acerca da possibilidade de o desporto representar um contexto promotor de desordens alimentares procurou verificar se existiam diferenças nas ocorrências destes problemas entre atletas e não atletas. Neste nível, existe ainda pouco consenso acerca do fato de o desporto representar um contexto promotor destes problemas. A título meramente de exemplo, alguns estudos indicam que os atletas são um grupo que evidencia maior prevalência de perturbações alimentares em relação aos não-atletas (ver Abrahan, 1996; Holm-Denoma, Scaringi, Gordon, Van Orden \& Joiner Jr., 2009), enquanto outros consideram o contrário (ver DiBartolo \& Shaffer, 2002; Madison \& Ruma, 2003). De forma inversa e em menor quantidade, existem ainda estudos que sugerem a possibilidade de o desporto representar um fator protetor para o desenvolvimento de desordens alimentares (ver Hausenblas \& McNally, 2004; Madison \& Ruma, 2003).

Numa análise global destes estudos, Byrne e McLean (2001) referem que existem mais estudos comprovativos da inexistência de diferenças entre atletas e não-atletas relativamente à prevalência de desordens alimentares. No entanto, este fato pode dever-se a problemas relacionados com o tamanho das amostras investigadas, à utilização de procedimentos de análise de dados inadequados, à variedade nos critérios de diagnóstico das desordens alimentares aplicados e até à diversidade de atletas estudados.

Algumas destas críticas e problemas levaram os autores a abandonarem esta tendência comparativa para passarem a analisar este tema exclusivamente no contexto desportivo. No entanto, e uma vez mais, os resultados encontrados são, em geral, bastante díspares.

Assim, Sundgot-Borgen (1994), num estudo bastante alargado, obteve resultados que indicaram mais de $15 \%$ das atletas de elite norueguesas com diagnóstico de uma desordem alimentar. Já Sanford-Martens e colaboradores (2005) relataram $5 \%$ dos atletas do sexo feminino e $2 \%$ dos atletas masculinos com diagnóstico de uma perturbação alimentar. Neste mesmo sentido, Beals e Hill (2006) examinaram a prevalência de desordens alimentares, disfunção menstrual e baixa 
densidade óssea em 112 atletas de desporto escolar. Destas atletas 25\% apresentaram diagnóstico de perturbação alimentar. Com valores muito inferiores, Petrie, Greenleaf, Carter e Reel (2007), numa investigação com 199 atletas masculinos, constataram que apenas $1 \%$ dos participantes poderiam ser diagnosticados com uma desordem alimentar e $16.6 \%$ como sintomáticos.

\section{Que atletas estaráo em risco?}

Nesta linha de estudo, procura-se analisar os problemas de comportamento alimentar tendo por base cada contexto desportivo e o tipo de atletas em causa. Neste sentido, e apesar da grande variabilidade de resultados, algumas investigaçôes têm vindo comprovar a prevalência destas problemáticas em atletas de algumas modalidades e/ou que realizam atividades com grande ênfase na aparência e forma físicas.

Segundo Byrne e McLean (2001), os primeiros trabalhos foram realizados na sua grande maioria com bailarinas. De um modo geral, comprovou-se que este grupo apresentava uma taxa elevada de desordens alimentares em relaçáo à população geral (Le Grange, Tibbs \& Noakes, 1994; Szmukler, Eisler, Gillies \& Hayward, 1985). Já na natação, Dummer, Rosen, Heusner, Roberts e Counsilman (1987) concluíram que uma significativa percentagem de atletas apresentava comportamentos alimentares de risco. Neste mesmo sentido, num estudo recente realizado por Ferrand, Magnan, Rouveix e Filaire (2007) com 33 adolescentes nadadores de elite verificou-se que todos apresentavam comportamentos alimentares de risco.

De um modo geral, estes resultados sustentam a ideia de que modalidades desportivas que se focalizam nos fatores estéticos e físicos, que enfatizam a magreza e formas corporais atléticas e apresentam exigências de peso corporal para categorias de competição podem aumentar o risco para as desordens alimentares. Isto mesmo tem vindo a ser demonstrado pelos estudos, constatando-se que os atletas que praticam desportos nos quais a magreza e peso corporal são particularmente decisivos para o sucesso desportivo estão mais expostos a estes problemas (Anshel, 2004; Beals \& Manore, 2000; Byrne e McLean, 2002; Petrie, 1996; Picard, 1999; Ringham et al., 2006; Zucker, Womble, Williamson \& Perrin, 1999).

Para além das possíveis diferenças entre modalidades desportivas, alguns autores têm dirigido a atenção para as questóes pessoais e desportivas no desenvolvimento das desordens alimentares (por exemplo, diferenças de sexo, índice de massa corporal, anos de prática e nível competitivo). 
Relativamente às diferenças em função do sexo, os estudos indicam que as mulheres apresentam maior risco de desenvolvimento de desordens alimentares (Haase, Prapavessis \& Owens, 2002; Hausenblas \& McNally, 2004; Petrie, 1996; Silva, Gomes \& Martins, 2011; Yates, Edman, Crago \& Crowell, 2003). A título ilustrativo, Petrie et al. (2007) investigaram a relação entre as desordens alimentares e os fatores de risco associados à imagem corporal, aos afetos negativos, à pressáo para o peso e para os comportamentos alimentares de risco em 199 atletas. Os autores concluíram que os atletas do sexo masculino têm comportamentos alimentares mais saudáveis, encontram-se moderadamente satisfeitos com os seus corpos e experimentam menor pressão para alterar o peso ou a composição corporal. Estes resultados sugerem que os fatores de risco para a populaçáo feminina, em geral, e para as atletas, em particular, podem não ser aplicados ao sexo masculino. De fato, enquanto as mulheres podem sentir maior pressáo associada à manutenção de um baixo peso, relacionando este fator a um melhor rendimento desportivo, os homens tendem a acreditar que aumentos no peso corporal traduzidos em maior massa muscular podem facilitar esse mesmo rendimento desportivo (Petrie \& Greenleaf, 2007).

Já o índice de massa corporal (IMC) indica-nos se um determinado indivíduo se encontra com peso baixo, peso normal, excesso de peso ou obesidade. Neste âmbito, os estudos de Petrie (1996) e De Bruin Karin, Oudejans e Bakker (2007) demonstraram que os atletas que apresentam valores mais baixos de IMC evidenciam maior probabilidade de desenvolverem uma perturbação alimentar. No entanto, a importância desta variável não foi evidente num estudo realizado por Ferrand, Champely e Filaire (2009) com atletas e não atletas franceses, tendo-se verificado que o IMC não se revelou uma variável preditora das desordens alimentares.

Quanto aos anos de prática desportiva existem poucos estudos sobre a sua importância na prevalência de desordens alimentares. Num dos poucos estudos realizados, DiBartolo e Shaffer (2002) encontraram uma correlação negativa entre a experiência desportiva dos atletas e os resultados obtidos nas escalas de comportamento alimentar.

No que se refere ao nível competitivo, algumas investigaçóes têm vindo indicar que à medida que aumentam as exigências desportivas criam-se mais condiçóes para o surgimento de problemas de comportamento alimentar (Thomas, Keel \& Heatherton, 2005; Silva et al., 2011). Por exemplo, Hausenblas e McNally (2004), num estudo com atletas de desporto escolar, universitário e de elite, analisaram a relaçáo entre o nível competitivo e os resultados obtidos no "Eating Disorder Inventory-2”. Os resultados demonstraram que os praticantes de desporto universitário apresentaram maiores pontuaçóes nas subescalas procura da magreza, bulimia, insatisfação corporal, ineficácia, mal-estar interoceptivo, medo 
da maturidade e insegurança social. Os atletas de elite obtiveram maiores resultados nas subescalas de perfecionismo e de desconfiança interpessoal. Neste mesmo sentido, De Bruin e colaboradores (2007) concluíram que ginastas de elite apresentaram maior frequência de comportamentos de dieta quando comparados com atletas que competiam num nível inferior.

\section{Quais as perspetivas futuras no estudo das desordens alimentares no desporto?}

Face à falta de resultados consensuais e/ou suficientes nas várias linhas de investigação apresentadas, Petrie e Greenleaf (2007) afirmaram que os estudos na área do desporto devem alterar o seu foco de interesse. Assim, a investigação poderá tornar-se mais útil se procurar analisar os fatores psicológicos associados às desordens alimentares nos atletas, uma vez que estes fatores representam riscos potenciais para o desenvolvimento destas perturbaçóes. Neste sentido, a compreensão dos fatores psicológicos associados ao desenvolvimento destes problemas pode representar um avanço significativo em termos da compreensão e intervenção neste tema. A título de exemplo, os atletas, principalmente de elite, são descritos como perfecionistas, orientados para os objetivos, competitivos e preocupados com o rendimento. Estas características estão associadas ao sucesso desportivo, mas também são facetas da personalidade que estão ligadas ao desenvolvimento das desordens alimentares (Hewitt, Flett \& Ediger, 1995). Assim sendo, torna-se necessário indicar quais os domínios mentais passíveis de estudo e que devem merecer a atenção dos autores interessados neste tema.

Embora seja extraordinariamente difícil decidir quais os domínios psicológicos que mais podem contribuir para a explicação das desordens alimentares, levantamos nesta última parte deste trabalho algumas áreas que têm merecido a atenção da investigação na Psicologia do Desporto mas que, curiosamente, têm tido pouca análise na compreensão dos comportamentos alimentares dos atletas.

Começando pelos processos de avaliação cognitiva, de acordo com o modelo transacional de Lazarus (1991), estes processos indicam-nos o modo como as pessoas percebem as situações de tensão que enfrentam (Lazarus \& Folkman, 1984). No domínio desportivo, existe evidência suficiente sobre a relação entre o modo como o atleta avalia as situaçóes e as respostas emocionais e comportamentais demonstradas (Rotella \& Lerner, 1992). Uma das emoçôes mais estudadas relaciona-se com a ansiedade. Neste caso, a investigação tem vindo demonstrar o impacto desta emoção no rendimento desportivo (Cruz, 1996; Edwards \& Hardy, 1996; GiacobbiJr. \& Weinberg, 2000), dando inclusivamente origem ao de- 
senvolvimento de modelos explicativos da ansiedade no desporto (Hardy, 1990; Smith, Smoll \& Schutz, 1990).

Apesar de estas indicaçóes sugerirem a importância da ansiedade no desporto, são poucos os estudos debruçados sobre a possível relação com as desordens alimentares. Por exemplo, Petrie e colaboradores (2007) constataram que os atletas com sintomatologia de desordens alimentares experienciavam maiores níveis de estresse do que o grupo de atletas assintomáticos. Por outro lado, Holm-Denoma e colaboradores (2009) concluíram que níveis elevados de ansiedade competitiva sáo preditores de sintomatologia bulímica e da vontade de ser magro. No entanto, sendo escassos os estudos neste domínio, torna-se muito difícil comprovar o verdadeiro impacto dos processos de avaliação cognitiva e das emoçóes (nas quais se inclui a ansiedade) nas desordens alimentares, sendo por isso fundamental desenvolver estudos que avaliem os efeitos destes processos psicológicos (Gomes, 2011).

No que se refere à orientação motivacional, o interesse pelo estudo desta dimensão em termos das desordens alimentares relaciona-se com a possível relação entre a forma como os atletas percebem o seu corpo e o tipo de resultados desportivos que pretendem obter. Por exemplo, nalguns desportos a vontade de manter um corpo magro e uma boa aparência física pode estar associada a melhores performances (De Bruin Karin, Oudejans \& Bakker, 2007).

De um modo geral, a investigação tem demonstrado que a orientação motivacional para o "ego" (na qual o objetivo é demonstrar um determinado nível de competência comparativamente a outras pessoas, sendo critério de sucesso a obtenção de um rendimento superior aos outros) está associada a reaçóes comportamentais e psicológicas mais negativas (por exemplo, menor comprometimento nos treinos, menor autoconfiança, menor satisfação e diversão no desporto e, pelo contrário, maior ansiedade competitiva, principalmente no caso dos atletas evidenciarem baixa perceção de competência) (Chi, 2004; Walling, Duda \& Chi, 1993). Pelo lado inverso, uma orientação para a tarefa (em que o objetivo é a melhoria pessoal, a aprendizagem e a mestria, representando critério de sucesso a capacidade da pessoa de progredir constantemente) aparece frequentemente relacionada com reaçóes mais adaptativas como, por exemplo, a motivação intrínseca, a perseverança e a satisfação (Roberts, 2001). Dada a natureza destas relaçóes, torna-se importante perceber a associação entre as desordens alimentares e a orientação motivacional, tendo por base abordagens teóricas explicativas da relação entre a motivação e a orientação seguida pelos atletas na formulação dos seus objetivos (Duda, 1992; Roberts, 2001). Num dos raros estudos realizados neste âmbito, De Bruin Karin, Bakker e Oudejans (2009) testaram as relaçóes entre as desordens alimentares em mulheres atletas de ginástica e de dança desportiva e as suas fontes de realização. 
Os resultados indicaram que uma maior orientação para o ego estava relacionada com maior frequência de comportamentos de dieta, maior nível de perfecionismo, maior pressão por parte dos pares relativamente ao peso e menor autoestima.

Outra dimensão passível de investigação refere-se aos processos de autoapresentação que, apesar da sua importância, ainda não adquiriram um estatuto relevante na explicação dos comportamentos de saúde (Ginis \& Leary, 2004). Tal como referem Leary, Tchividjian e Kraxberger (1994), os motivos interpessoais (nos quais se inclui a autoapresentação) são por vezes contemplados nos modelos de comportamentos de saúde como estando relacionados com os valores pessoais, os resultados obtidos e as barreiras à mudança comportamental, mas o seu papel específico na saúde está praticamente por identificar.

Neste sentido, a autoapresentação (ou gestão da impressão, como é por vezes designada) refere-se aos processos pelos quais as pessoas tentam monitorizar e controlar a forma como são percebidas e avaliadas pelos outros (Leary, 1994). Aplicando esta conceitualização da autoapresentação ao domínio dos comportamentos alimentares, as pessoas também podem assumir uma vontade de parecerem atrativas aos outros ou, pelo menos, evitarem ser vistas como pouco atraentes. Do ponto de vista da investigação, existe alguma evidência acerca da importância dos processos de autoapresentaçáo nos comportamentos alimentares, principalmente nas mulheres que assumem maior necessidade de aprovação social e de transmitirem uma impressão de ser feminina (ver Mori, Chaiken \& Pliner, 1987; Pliner \& Chaiken, 1990). Apesar da importância que este tema tem na psicologia do desporto (Prapavessis, 2004), são ainda escassos os estudos debruçados sobre a relação entre os processos de autoapresentação e as desordens alimentares, tanto mais se tivermos em conta que foi em 1992 que Mark Leary publicou um dos primeiros trabalhos a chamar a atenção para o papel da autoapresentação em contextos desportivos e de prática de exercício físico (Leary, 1992).

Do ponto de vista da relação treinador e atletas, existem indicações de que atletas com desordens alimentares, particularmente com anorexia nervosa, parecem mais propensos para o conformismo e para a necessidade de aprovação (Garfinkel \& Garner, 1982). Estes atletas apresentam uma grande vontade de agradar bem como algum receio de desapontar as outras pessoas (Thompson \& Sherman, 1999). Por isso mesmo, é bastante frequente a existência de um relacionamento positivo entre atletas e treinadores, não sendo habitual os responsáveis técnicos considerarem que os atletas têm um problema com a alimentação. Este fator pode levar a uma maior dificuldade em sinalizar sinais de perigo nestes atletas quando estes efetivamente apresentam uma desordem alimentar. Neste sentido, estudar os contributos teóricos atualmente existentes acerca dos comportamentos dos treinadores e dos estilos de liderança assumidos na relação com os atletas (Chelladurai, 2007) pode representar um avanço 
na compreensão dos comportamentos alimentares dos atletas. Tal como referem Biesecker e Martz (1999), torna-se fundamental determinar se os comportamentos dos treinadores têm realmente um impacto na incidência das desordens alimentares, pois só assim poderão ser mobilizados esforços no sentido de prevenir eventuais efeitos indesejados da influência destes profissionais. Esta linha de investigaçáo parece ser muito promissora, existindo autores que sugerem uma relação entre os comentários depreciativos dos treinadores acerca da aparência física e corporal dos atletas e a tendência destes de usarem métodos de controle de peso pouco saudáveis e evidenciarem sintomas de desordens alimentares (Kerr, Berman \& De Souza, 2006). A título de exemplo, Muscat e Long (2008) realizaram um estudo com mulheres atletas e praticantes de exercício físico no sentido de verificar a influência que os comentários críticos dos outros poderiam produzir na tendência para as desordens alimentares. Neste conjunto de indivíduos que poderiam emitir comentários críticos estavam incluídos os treinadores e os monitores de exercício físico, tendo-se verificado que as mulheres que relataram mais comentários críticos acerca do seu peso e forma corporal demonstraram maiores problemas relacionados com os comportamentos alimentares.

Finalmente, a investigação neste domínio pode se beneficiar da inclusão de medidas acerca da satisfação dos atletas com o seu corpo e forma física. Esta faceta torna-se importante pois existem dados que sugerem que os atletas (particularmente as mulheres) sentem-se insatisfeitos com o seu corpo quando constatam discrepâncias entre o modo como se avaliam e a forma como as outras pessoas os poderão avaliar fisicamente (Stice, Chase, Stormer \& Appel, 2001). A título de exemplo, Petrie e colaboradores (2007) verificaram que os atletas com sintomatologia relacionada com as desordens alimentares reportaram maior medo de engordar e a sua autoestima era influenciada pelo peso. Além disso, estes atletas apresentaram maior flutuação no peso do que o grupo de atletas assintomáticos. Neste sentido, é de se levantar a hipótese de uma relação entre a insatisfação corporal e as desordens alimentares, devido à adoção de dietas e restriçóes alimentares e ao surgimento do humor negativo (Smith \& Petrie, 2008).

\section{A teoria pode ajudar a compreender as desordens alimentares no desporto?}

Numa tentativa de integração teórica destes dados da investigação, Petrie e Greenleaf (2007) propuseram um modelo etiológico da relação entre os fatores de risco e o desenvolvimento de desordens alimentares no desporto. Assim, para além das dimensóes psicológicas que acabamos de referir, os autores assinalam a 
importância de outras variáveis como, por exemplo, a autoestima, o autoconceito, o "locus" de controle, o neuroticismo, o perfecionismo, o autoconceito físico e o controle da impulsividade. Em termos gerais, o modelo preconiza uma relação entre alguns fatores antecedentes (pressóes sociais e desportivas para magreza e forma física) que, por sua vez, influenciam a internalização de um ideal corporal magro e atlético. Esta idealização pode acabar por levar a sentimentos de insatisfação corporal, promovendo uma emocionalidade negativa. Como forma de lidar com este mal-estar, os atletas tendem a assumir comportamentos de restrição alimentar, que também podem ser modelados (ou influenciados) por pessoas significativas (ex.: treinador, pais, colegas de equipe, etc.). $\mathrm{O}$ resultado final deste processo é o desenvolvimento de desordens alimentares.

Dito de um modo mais simples, a relação entre as pressóes sociais e desportivas e o desenvolvimento de problemas alimentares no desporto é mediada por um conjunto de variáveis (por exemplo, processos de internalização, insatisfação corporal, afetos negativos, comportamentos de restrição e modelagem de comportamentos) que, no seu conjunto, representam fatores de risco para o desenvolvimento de problemas graves do ponto de vista alimentar nas populações desportivas.

Dada a formulação recente do modelo, faltam ainda estudos comprovativos acerca da sua validade na aplicaçáo aos diversos contextos desportivos. No entanto, e como referem os próprios autores, se a investigaçáo futura procurar testar as relaçóes entre as variáveis propostas, poderemos determinar a razão de os atletas estarem em risco de desenvolvimento de desordens alimentares e até que ponto estes fatores de risco estão presentes nas mais variadas modalidades desportivas.

Além da importância de testar modelos teóricos na compreensão das desordens alimentares, torna-se igualmente importante estabelecer se estes conceitos podem ser utilizados para o desenvolvimento de programas de prevenção e intervenção sobre os comportamentos alimentares de risco dos atletas. Uma vez mais, existe uma escassez de informaçôes sobre este tema. No entanto, existem indicaçôes da literatura que sugerem a importância de prestar particular importância ao modo como os atletas avaliam o seu peso corporal pois discrepâncias entre o seu peso real e o peso ideal podem contribuir para as desordens alimentares (Gomes, Martins \& Silva, 2011). Por outro lado, tal como sugerido neste trabalho, existem fatores psicológicos que podem estar particularmente associados aos comportamentos alimentares desajustados (ex.: processos de avaliação cognitiva, níveis de ansiedade, insatisfação corporal, etc.), tornando-se agora relevante verificar se intervençóes psicológicas que promovam maior bem-estar nestes domínios poderão prevenir a ocorrência de problemas no nível alimentar. 
Em síntese, apesar do interesse da investigação centrada na análise da prevalência das desordens alimentares no desporto, parece existir evidência suficiente para a necessidade do estudo das variáveis psicológicas associadas a este problema. Esta "nova" abordagem do estudo das desordens alimentares pode facilitar decisivamente o trabalho dos técnicos que trabalham com os atletas, direcionando a sua intervenção no sentido de promover competências psicológicas que ajudem os praticantes a resistirem aos efeitos menos positivos do desporto, nos quais se incluem os comportamentos alimentares de risco.

\section{Referências}

Abraham, S. (1996). Eating and weight controlling behaviours of young ballet dancers. Psychopathology, 29(4), 218-222.

American Psychiatric Association. (1994). Diagnostic and statistical manual of mental disorders (4th ed.). Washington, DC: Author.

American Psychiatric Association. (2000). Diagnostic and statistical manual of mental disorders (4th ed., text revision). Washington, DC: Author.

American Psychiatric Association (2002). Manual de diagnóstico e estatistica de distúrbios mentais Texto Revisto (4a ed.) (DSM-IV-TR). Lisboa: Climepsi editores. (Publicação original 2000).

Anshel, M. H. (2004). Sources of disordered eating patterns between ballet dancers and non-dancers. Journal of Sport Behavior, 27, 115-133.

Beals, K. A. \& Hill, A. K. (2006). The prevalence of disordered eating, menstrual dysfunction, and low bone mineral density among US collegiate athletes. International Journal of Sport Nutrition and Exercise Metabolism, 16, 1-23.

Beals, K. A. \& Manore, M. M. (2000). Behavioral, psychological, and physical characteristics of female athletes with subclinical eating disorders. International Journal of Sport Nutrition and Exercise Metabolism, 10, 128-143.

Biesecker, A. C. \& Martz, D. M. (1999). Impact of coaching style on vulnerability for eating disorders: An analog study. Eating Disorders, 7, 235-244.

Byrne, S. \& McLean, N. (2001). Eating disorders in athletes: a review of the literature. Journal of Science and Medicine in Sport, 4(2), 145-159.

Byrne, S. \& McLean, N. (2002). Elite athletes: effects of the pressure to be thin. Journal of Science and Medicine in Sport, 5, 80-94.

Chelladurai, P. (2007). Leadership in sports. In: G. Tenenbaum \& R. C. Eklund (Eds.). Handbook of sport psychology (3rd ed., pp. 113-135). Hoboken, New Jersey: John Wiley e Sons.

Chi, L. (2004). Achievement goal theory. In: T. Morris \& J. Summers (Eds.). Sport psychology: theory, applications and issues (2nd ed.) (pp. 152-174). Austrália: John Wiley e Sons. 
Cogan, K. D. (2005). Eating disorders: when rations become irrational. In: S. Murphy (Ed.). The sport psych handbook (pp. 237-256). Champaign, IL: Human Kinetics Publishers.

Cruz, J. F. (1996). A relação entre ansiedade e rendimento desportivo: teorias e hipóteses explicativas. In: J. F. Cruz (Ed.). Manual de psicologia do desporto (pp. 215-263). Braga: SHO-Sistemas Humanos e Organizacionais.

De Bruin, Karin A. P., Bakker, F. C. \& Oudejans, R. R. D. (2009). Achievement goal theory and disordered eating: Relationships of disordered eating with goal orientations and motivational climate in female gymnasts and dancers. Psychology of Sport and Exercise, 10, 72-79.

De Bruin, Karin A. P; Oudejans, R. R. D. \& Bakker, F. C. (2007). Dieting and body image in aesthetic sports: a comparison of Dutch female gymnasts and nonaesthetic sport participants. Psychology of Sport and Exercise, 8, 507-520.

DiBartolo, P. M. \& Shaffer, C. (2002). A comparison of female college athletes and nonathletes: eating disorder symptomatology and psychological well-being. Journal of Sport e Exercise Psychology, 24, 33-41.

Drinkwater, B. L.; Loucks, A.; Sherman, R. T.; Sundgot-Borgen, J. \& Thompson, R. A. (2005). Position stand on the female athlete triad. IOC Medical Commission Working Group Women in Sport. Recuperado em 4 fevereiro, 2012 de <http://multimedia.olympic.org/ en_report_917.pdf>.

Duda, J. L. (1992). Motivation in sport settings: A goal perspective approach. In: G. C. Roberts (Ed.). Motivation in sport and exercise (pp. 47-91). Champaign, IL: Human Kinetics.

Dummer, G. M.; Rosen, L. W.; Heusner, W. W.; Roberts, P. J. \& Counsilman, J. E. (1987). Pathogenic weight-control behaviours of young competitive swimmers. The Physician and Sports Medicine, 15(5), 75-84.

Edwards, T. \& Hardy, L. (1996). The interactive effects of intensity and direction of cognitive and somatic anxiety, and self-confidence upon performance. Journal of Sport e Exercise Psychology, 18, 296-312.

Fairburn, C. G. (1997). Eating disorders. In: D. M. Clark \& C. G. Fairburn (Eds.). Science and Practice of Cognitive Behaviour Therapy (pp. 209-243). New York: Oxford University Press.

Ferrand, C.; Champely, S. \& Filaire, E. (2009). The role of body-esteem in predicting disordered eating symptoms: a comparison of French aesthetic athletes and non-athletic females. Psychology of Sport and Exercise, 10, 373-380.

Ferrand, C.; Magnan, C.; Rouveix, M. \& Filaire, E. (2007). Disordered eating, perfectionism and body-esteem of elite synchronized swimmers. European Journal of Sport Science, 7(4), 223-230.

Garfinkel, P. E. \& Garner, D. M. (1982). Anorexia nervosa: a multidimensional perspective. New York: Brunner-Mazel.

Giacobbi Jr., P. R. \& Weinberg, R. S. (2000). An examination of coping in sport: individual trait anxiety differences and situational consistency. The Sport Psychologist, 14, 42-62. 
Ginis, K. A. M. \& Leary, M. R. (2004). Self-presentational processes in health-damaging behaviour. Journal of Applied Sport Psychology, 16, 59-74.

Gomes, A. R. (2011). Adaptação humana em contextos desportivos: contributos da teoria para a avaliação psicológica. Avaliação Psicológica, 10(1), 13-24.

Gomes, A. R.; Martins, C. \& Silva, L. (2011). Eating disordered behaviours in Portuguese athletes: the influence of personal, sport, and psychological variables. European Eating Disorders Review, 19(3), 190-200.

Haase, A. M.; Prapavessis, H. \& Owens, R. G. (2002). Perfectionism, social physique anxiety and disordered eating: a comparison of male and female elite athletes. Psychology of Sport and Exercise, 3, 209-222.

Hardy, L. (1990). A catastrophe model of performance in sport. In: J. G. Jones \& L. Hardy (Eds.). Stress and performance in sport (pp. 81-106). Chichester, UK: Wiley.

Hausenblas, H. A. \& McNally, K. D. (2004). Eating disorder prevalence and symptoms for track and field athletes and nonathletes. Journal of Applied Sport Psychology, 16, 274-286.

Hewitt, P. L.; Flett, G. L. \& Ediger, E. (1995). Perfectionism traits and perfectionistic self-presentation in eating disorders attitudes. Characteristics and symptoms. International Journal of Eating Disorders, 18, 317-326.

Holm-Denoma, J. M.; Scaringi, V.; Gordon, K. H.; Van Orden, K. A. \& Joiner Jr., T. E. (2009). Eating disorder symptoms among undergraduate varsity athletes, club athletes, independent exercisers, and nonexercisrs. International Journal of Eating Disorders, 42, 47-53.

Kerr, G.; Berman, E. \& De Souza, M. (2006). Disordered eating in women's gymnastics: perspectives of athletes, coaches, parents and judges. Journal of Applied Sport Psychology, 18, 28-43.

Lazarus, R. S. (1991). Emotion and adaptation. New York: Oxford University Press.

Lazarus, R. S. \& Folkman, S. (1984). Stress, appraisal and coping. New York: Springer.

Le Grange, D.; Tibbs, J. \& Noakes, T. D. (1994). Implications of a diagnosis of anorexia nervosa in a ballet school. International Journal of Eating Disorders, 15(4), 369-376.

Leary, M. R. (1992). Self-presentation processes in exercise and sport. Journal of Sport e Exercise Psychology, 14, 339-351.

Leary, M. R. (1994). Self-presentation: impression management and social behavior. Dubuque, IA: Brown e Benchmark.

Leary, M. R.; Tchividjian, L. R. \& Kraxberger, B. E. (1994). Self-presentation can be hazardous to your health: impression management and health risk. Health Psychology, 13, 461-470.

Madison, J. K. \& Ruma, S. L. (2003). Exercise and athletic involvement as moderators of severity in adolescents with eating disorders. Journal of Applied Sport Psychology, 15, 213-222.

Morgan, W. P. (1979). Negative addiction in runners. Physican and Sports Medicine, 7, 57-70.

Mori, D.; Chaiken, S. \& Pliner, P. (1987). "Eating lightly" and the self-presentation of femininity. Journal of Personality and Social Psychology, 53, 693-702.

Psic. Clin., Rio de Janeiro, vol. 24, N.I I, P. 83 - IOO, 2012 
Muscat, A. C. \& Long, B. C. (2008). Critical comments about body shape and weight: Disordered eating of female athletes and sport participants. Journal of Applied Sport Psychology, 20, 1-24.

Otis, C. L.; Drinkwater, B.; Johnson, M.; Loucks, A. \& Wilmore, J. (1997). American College of Sports Medicine position stand: the female athlete triad. Medicine and Science in Sports and Exercise, 29, 1-9.

Petrie, T. A. (1996). Differences between male and female college lean sport athletes, nonlean sport athletes, and non-athletes on behavioral and psychological indices of eating disorders. Journal of Applied Sport Psychology, 8, 218-230.

Petrie, T. A. \& Greenleaf, C. (2007). Eating disorders in sport: from theory to research to intervention. In: G. Tenenbaum \& R. Eklund (Eds.). Handbook of sport psychology (3rd ed.) (pp. 352-378). Hoboken, NJ: Wiley.

Petrie, T. A.; Greenleaf, C.; Carter, J. E. \& Reel, J. J. (2007). Psychosocial correlates of disordered eating among male collegiate athletes. Journal of Clinical Sport Psychology, 1, 340-357.

Picard, C. (1999). The level of competition as a factor for the development of eating disorders in female collegiate athletes. Journal of Youth and Adolescence, 28, 583-594.

Pliner, P. \& Chaiken, S. (1990). Eating, social motives, and selfpresentation in women and men. Journal of Experimental Social Psychology, 26, 240-254.

Prapavessis, H. (2004). Introduction to self-presentation in exercise and sport. Journal of Applied Sport Psychology, 16, 1-2.

Raich, R. (2001). Anorexia e bulimia. Amadora: McGraw-Hill.

Ricciardelli, L. A. \& McCabe, M. P. (2001). Child body image concerns and eating disturbance: a review of the literature. Clinical Psychology Review, 21(3), 325-344.

Ringham, R.; Klump, K.; Kaye, W.; Stone, D.; Libman, S.; Stowe, S. et al. (2006). Eating disorder symptomatology among ballet dancers. International Journal of Eating Disorders, 39, 503-508.

Roberts, G. C. (2001). Understanding the dynamics of motivation in physical activity: the influence of achievement goals on motivational processes. In: G. C. Roberts (Ed.). Advances in motivation in sport and exercise (pp. 1-51). Champaign, IL: Human Kinetics.

Rotella, R. J. \& Lerner, J. D. (1992). Responding to competitive pressure. In: T. Horn (Ed.). Advances in sport psychology (pp. 528-541). Champaign, IL: Human Kinetics.

Sanford-Martens, T. C.; Davidson, M. M.; Yakushko, O. F.; Martens, M. P.; Hinton, P. \& Beck, N. (2005). Clinical and subclinical eating disorders: an examination of collegiate athletes. Journal of Applied Sport Psychology, 17, 79-86.

Schur, E.; Sanders, M. \& Steiner, H. (2000). Body dissatisfaction and dieting in young children. International Journal of Eating Disorders, 27, 74-82.

Silva, L.; Gomes, A. R. \& Martins, C. (2011). Psychological factors related to eating disorders: a study with Portuguese athletes. The Spanish Journal of Psychology, 14(1), 323-335. Recuperado em 4 janeiro, 2012 de <http://hdl.handle.net/1822/12220>.

Smith, A. \& Petrie, T. (2008). Reducing the risk of disordered eating among female athletes: a test of alternative interventions. Journal of Applied Sport Psychology, 20, 392-407. 
Smith, R. E.; Smoll, F. L. \& Schutz, R. W. (1990). Measurement and correlates of sport-specific cognitive and somatic trait anxiety: the Sport Anxiety Scale. Anxiety Research, 2, 263-280.

Stice, E.; Chase, A.; Stormer, S. \& Appel, A. (2001). A randomized trial of a dissonance-based eating disorder prevention program. International Journal of Eating Disorders, 29, 247-262.

Sudi, K.; Öttl, K.; Payerl, D.; Baumgartl, P.; Tauschmann, K. \& Müller, W. (2004). Anorexia athletica. Nutrition, 20, 657-661.

Sundgot-Borgen, J. (1993). Prevalence of eating disorders in elite female athletes. International Journal of Sports Nutrition, 3, 29-40.

Sundgot-Borgen, J. (1994). Risk and trigger factors for the development of eating disorders in female elite athletes. Medicine e Science in Sports e Exercise, 26(4), 414-419.

Sundgot-Borgen, J. \& Torstveit, M. K. (2004). Prevalence of eating disorders in elite athletes is higher than in the general population. Clinical Journal of Sports Medicine, 14, 25-32.

Szmukler, G. I.; Eisler, I.; Gillies, C. \& Hayward, M. E. (1985). The implications of anorexia nervosa in a ballet school. Journal of Psychiatric Research, 19, 177-181.

Thomas, J. J., Keel, P. K. \& Heatherton, T. F. (2005). Disordered eating attitudes and behaviors in ballet students: examination of environmental and individual risk factors. International Journal of Eating Disorders, 38, 263-268.

Thompson, R. A. \& Sherman, R. (1993). Helping athletes with eating disorders. Champaign, IL: Human Kinetics.

Thompson, R. A. \& Sherman, R. T. (1999). "Good athlete” traits and characteristics of anorexia nervosa: are they similar? Eating Disorders, 7, 181-190.

Walling, M.; Duda, J. \& Chi, L. (1993). The perceived motivational climate in sport questionnaire: construct and predictive validity. Journal of Sport e Exercise Psychology, 15, 172-183.

Yates, A.; Edman, J. D.; Crago, M. \& Crowell, D. (2003). Eating disorder symptoms in runners, cyclists, and paddlers. Addictive Behavior, 28, 1473-1480.

Zucker, N. L.; Womble, L. G.; Williamson, D. A. \& Perrin, L. A. (1999). Protective factors for eating disorders in female college athletes. Eating Disorders, 7, 207-218.

\section{Nota}

1 Este trabalho resulta parcialmente da tese de Mestrado em Psicologia, Especialização em Psicologia Desportiva realizada pelo primeiro autor deste artigo no Instituto de Educação e Psicologia, Universidade do Minho (Braga, Portugal).

Recebido em 17 de fevereiro de 2012 Aceito para publicação em 24 de julho de 2012 